\title{
On the fine structure of sunspot penumbrae
}

\section{The nature of the Evershed flow}

\author{
J. M. Borrero ${ }^{1, \star}$, A. $\operatorname{Lagg}^{1}$, S. K Solanki ${ }^{1}$, and M. Collados ${ }^{2}$ \\ 1 Max-Planck Institut für Sonnensystemforschung, 37191 Katlenburg-Lindau, Germany \\ e-mail: borrero@ucar.edu, lagg@mps.mpg.de, solanki@mps.mpg.de \\ 2 Instituto de Astrofísica de Canarias, 38200, Vía Láctea s/n, La Laguna, Tenerife, Spain \\ e-mail: mcv@iac.es
}

Received 16 December 2004 / Accepted 4 February 2005

\begin{abstract}
We investigate the fine structure of the sunspot penumbra by means of a model that allows for a flux tube in horizontal pressure balance with the magnetic background atmosphere in which it is embedded. We apply this model to spectropolarimetric observations of two neutral iron lines at $1.56 \mu \mathrm{m}$ and invert several radial cuts in the penumbra of the same sunspot at two different heliocentric angles. In the inner part of the penumbra we find hot flux tubes that are somewhat inclined to the horizontal. They become gradually more horizontal and cooler with increasing radial distance. This is accompanied by an increase in the velocity of the plasma and a decrease of the gas pressure difference between flux tube and the background component. At large radial distances the flow speed exceeds the critical speed and evidence is found for the formation of a shock front. These results are in good agreement with simulations of the penumbral fine structure and provide strong support for the siphon flow as the physical mechanism driving the Evershed flow.
\end{abstract}

Key words. Sun: sunspots - line: profiles - Sun: magnetic fields - Sun: infrared

\section{Introduction}

The picture of the magnetic fine structure of the penumbra has strongly evolved over the last decade (e.g., Degenhardt \& Wiehr 1991; Title et al. 1993; Solanki \& Montavon 1993; Rimmele 1995; Stanchfield et al. 1997; Westendorp Plaza et al. 1997; Schlichenmaier et al. 1998a; Scharmer et al. 2002; see Solanki 2003, Bellot Rubio 2003 and Thomas \& Weiss 2004, for an overview). It is now accepted that the penumbral magnetic field is uncombed, i.e. inclined at least in two different directions on a small scale. There is also considerable evidence that the more horizontal component must be in the form of flux tubes, although the diameter of these flux tubes is still a matter of debate (Sánchez Almeida 1998, 2001; Martínez Pillet 2000, 2001). These flux tubes carry the Evershed flow (Evershed 1909; Title et al. 1993; Westendorp Plaza et al. 2001a,b; Bellot Rubio et al. 2003, 2004; Borrero et al. 2004). Many of the tubes return to the solar interior within the penumbra (Westendorp Plaza et al. 1997; del Toro Iniesta et al. 2001; Mathew et al. 2003; Borrero et al. 2004) and along with the magnetic flux a large fraction of the mass flux carried by the Evershed flow returns also to the solar interior within the penumbra or just outside it (Börner \& Kneer 1992; Solanki et al. 1994, 1999).

^ Present address: High Altitude Observatory, 3450 Mitchell Lane, 80301 Boulder, Colorado, USA.
The combination of these results raises questions regarding the commonly considered physical mechanism driving the Evershed flow. Since the wave hypothesis is ruled out (Bünte \& Solanki 1995) and episodic Evershed flow produced when a flux tube falls and drains (Wentzel 1992) faces difficulties due to the relative immutability of the penumbral magnetic pattern (Solanki \& Rüedi 2003), the most widely accepted mechanism is that the flow is caused by a gas pressure gradient between the upflowing and the downflowing footpoints, also referred to as the siphon flow mechanism (Meyer \& Schmidt 1968; see also Thomas 1988). Commonly, this pressure gradient is thought to be produced by a difference in the field strength between the footpoints which, due to horizontal pressure balance, leads to a gas pressure difference (e.g. Degenhardt 1989, 1991; Thomas \& Montesinos 1991, 1993; Montesinos \& Thomas 1997). All else being equal, the gas flows from the footpoint with lower field strength to the footpoint with the higher field strength. However, if most of the gas flows only within the penumbra, then due to the roughly factor of 2 larger magnetic field strength at the inner boundary of the penumbra compared to the outer edge, one would naively expect the gas to flow inwards, contradicting observations. Montesinos \& Thomas (1997) have argued that this radial decrease of the field strength is only apparent, being caused by different $\tau=1$ levels at the footpoints and the fact that local intense magnetic flux concentrations at the outer penumbral edge could not be easily resolved. 
A possible resolution of this dilemma was noticed by Borrero et al. (2004, hereafter Paper I), who found that whereas the strength of the inclined magnetic component drops very rapidly in the radial direction, as required by a global magnetic structure close to potential (see Jahn 1989), the horizontal component carrying the Evershed flow shows far less variation (cf. Rüedi et al. 1998, 1999). The analysis in Paper I was incomplete in the sense that the two components were independent of each other. Here we overcome this shortcoming and take into account the flux-tube structure of the field and the pressure balance between components. We apply a powerful inversion technique (described in Sect. 2) to spectropolarimetric observations of infrared Zeeman sensitive spectral lines (Sect. 3). General results are presented and discussed in Sect. 4. In Sect. 5 we discuss the generation of Net Circular Polarization by the uncombed model and the implications for the typical size of the penumbral flux tubes. Section 6 describes our results in the framework of the theoretical models employed to explain the Evershed effect in terms of gas pressure differences. Our main findings and conclusions are summarized in Sect. 7.

\section{Description of the penumbral model and Stokes profile inversion}

The analysis of spectropolarimetric observations of the sunspot penumbra by means of Stokes profile inversion has, so far, either considered 2 distinct components (assuming the physical quantities to be constant with depth: Bellot Rubio et al. 2003, 2004) or 1 component (allowing gradients to be present; Westendorp Plaza et al. 1997, 2001a,b; Bellot Rubio et al. 2002; Mathew et al. 2003). In Paper I we suggested that the uncombed penumbral model described by Solanki \& Montavon (1993) provides a picture for the penumbral fine structure that is able to encompass the results of these investigations. This conclusion was based on the application of a Stokes profile inversion technique assuming the two different geometries mentioned above, together with considerations on how the area asymmetry of the circular polarization profiles is influenced by gradients in the magnetic and kinematic stratifications.

In this paper we carry out Stokes profile inversions based on the uncombed model. This consists of a flux tube embedded in a magnetic surrounding atmosphere. The forward modelling for the considered geometry has already been addressed (see e.g. Degenhardt \& Kneer 1992; Solanki \& Montavon 1993; Martínez Pillet 2000). The basic geometry is illustrated in Fig. 1. The simplest representation of the uncombed model is in terms of two rays: the first ray passes along the surrounding atmosphere (vertical dot-dashed line pointing towards the observer) while the other ray cuts both surrounding atmosphere and the flux tube (vertical dashed line) ${ }^{1}$. Let us denote with $\chi_{\mathrm{s}}$ any of the magnetic and kinematic physical quantities

\footnotetext{
${ }^{1}$ Note that this geometrical simplification in terms of two rays implies that we are considering the cross section of the flux tube to be square instead of circular as in Fig. 1.
}

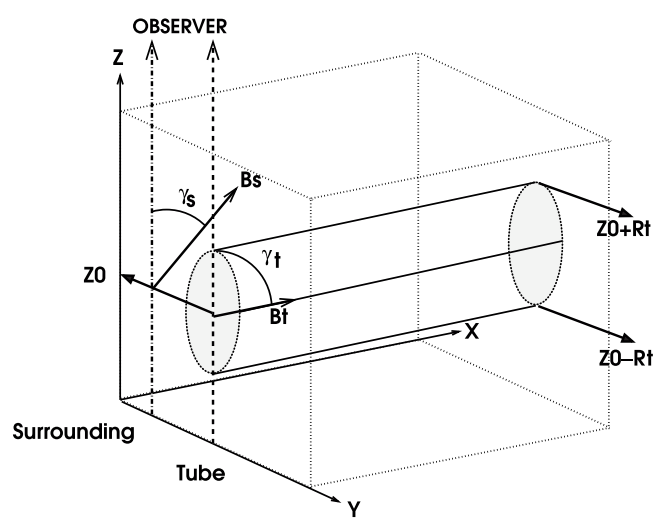

Fig. 1. Example of the geometrical scheme used in the inversion. The radiative transfer equation is solved along the 2 rays (dashed and dotdashed lines) representing the embedded flux tube and surrounding atmosphere respectively. $\gamma_{\mathrm{s}}$ and $\gamma_{\mathrm{t}}$ refer to the inclination of the magnetic field vector with respect to the observer. In this picture for simplicity the heliocentric angle is $\theta=0$ and $\gamma_{\mathrm{t}}=90^{\circ}$.

for the surrounding atmosphere ${ }^{2}$. For the flux tube component we adopt the following form for $\chi_{\mathrm{t}}(z)$ :

$\chi_{\mathrm{t}}(z)= \begin{cases}\chi_{\mathrm{t}} & \text { if } z \in\left[z_{0}-R_{\mathrm{t}}, z_{0}+R_{\mathrm{t}}\right] \\ \chi_{\mathrm{s}} & \text { otherwise }\end{cases}$

where $\chi_{\mathrm{t}}$ on the right hand side of Eq. (1) is height independent, $z_{0}$ is the height where the axis of the flux tube is located and $R_{\mathrm{t}}$ is its radius. Note that Eq. (1) implies that physical stratifications along the flux tube atmosphere (vertical dashed line in Fig. 1) are the same as in the surrounding atmosphere above and beneath the flux tube: $z<z_{0}-R_{\mathrm{t}}$ and $z>z_{0}+R_{\mathrm{t}}$. At the flux tube lower and upper boundaries $\left(z^{*}=z_{0} \pm R_{\mathrm{t}}\right)$ the physical quantities suffer a jump whose magnitude is $\Delta \chi=\chi_{\mathrm{t}}-\chi_{\mathrm{s}}$. These jumps/gradients are the essential ingredients to explain the Net Circular Polarization (NCP) observed in the sunspot penumbra (Solanki \& Montavon 1993; cf. Martínez Pillet 2000).

The inversions based on this geometry have been carried out using the inversion code SPINOR (see Frutiger et al. 1999; Frutiger 2000). The code performs spectral line synthesis in Local Thermodynamic Equilibrium (LTE) and employs a Levenberg-Marquart nonlinear least-squares $\chi^{2}$ minimization algorithm (Press et al. 1986), whereby derivatives are calculated through numerical response functions (RFs; see Ruiz Cobo \& del Toro Iniesta 1992). The free parameters allowed in the inversion are, for the surrounding atmosphere: $V_{\mathrm{LOS}, \mathrm{s}}$ (line of sight velocity), $B_{\mathrm{s}}$ (magnetic field strength), $\gamma_{\mathrm{s}}$ (magnetic field inclination with respect to the observer), $\phi_{\mathrm{s}}$ (magnetic field azimuth) and $T_{\mathrm{s}}\left(\tau_{5}=1\right.$ ) for temperature, where $\tau_{5}$ is the optical depth at a reference wavelength of $5000 \AA$. The height dependence of $T_{\mathrm{s}}(\tau)$ is taken from the penumbral model by del Toro Iniesta et al. (1994). When $T_{\mathrm{s}}\left(\tau_{5}=1\right)$ is changed by $\Delta T$ the temperature at all heights is changed additively: $T_{\mathrm{s}}(\tau)=T_{\mathrm{s} \text {,old }}(\tau)+\Delta T$.

For the flux tube the free parameters are: $V_{\mathrm{LOS}, \mathrm{t}}, B_{\mathrm{t}}, \gamma_{\mathrm{t}}$, $\phi_{\mathrm{t}}$, macro and microturbulent velocities, $v_{\text {mac,t }}$ and $v_{\text {mic,t }}$, and $T_{\mathrm{t}}\left(\tau_{5}=1\right)$ (where again $T_{\mathrm{t}}(\tau)$ is taken from the mean penumbra

\footnotetext{
${ }^{2} \chi_{\mathrm{s}}$ is assumed to be height independent.
} 
model of del Toro Iniesta et al.). In addition, $z_{0}, R_{\mathrm{t}}$ and $\alpha_{\mathrm{t}}$ (fractional horizontal area covered by the flux tube component with respect to the total area) are also allowed to change. Finally we employ a stray light correction to model the contribution of light from the neighbouring granulation that enters into the spectrograph's slit. To this end we used the two component model for the quiet sun from Borrero \& Bellot Rubio (2002) to produce synthetic intensity profiles, $I_{\mathrm{q}}$, of the observed spectral lines (see Sect. 3) and combined it with the emergent spectrum of the pure penumbral profiles using a filling factor $\alpha_{\mathrm{q}}$ which is also retrieved from the inversion. This results in a total of 16 free parameters. This compares positively with the inversions carried out in Paper I, which used a total number of 23 and 18 free parameters for the 1 component and 2 component inversions, respectively.

The radiative transfer equation is integrated using the Hermitian Approach (see Bellot Rubio et al. 1998) for each ray separately. The Stokes profiles from quiet sun, flux tube and surrounding atmosphere are finally combined using the filling factors $\alpha_{\mathrm{t}}$ and $\alpha_{\mathrm{q}}$ :

$\boldsymbol{S}(\lambda)=\alpha_{\mathrm{q}} \boldsymbol{S}_{\mathrm{q}}(\lambda)+\left(1-\alpha_{\mathrm{q}}\right)\left[\left(1-\alpha_{\mathrm{t}}\right) \boldsymbol{S}_{\mathrm{s}}(\lambda)+\alpha_{\mathrm{t}} \boldsymbol{S}_{\mathrm{t}}(\lambda)\right]$,

where $S$ represents the Stokes vector $(I, Q, U, V)$ : as arising from the surrounding atmosphere (dot-dashed ray in Fig. 1) $S_{\mathrm{s}}$, from the ray cutting the flux tube (dashed ray in Fig. 1) $S_{t}$, as well as the quiet sun contribution $S_{\mathrm{q}}=\left(I_{\mathrm{q}}, 0,0,0\right)$ which is a non polarized contribution and only affects the total intensity profiles. These synthetic profiles are now compared with the observations and the free parameters are changed according to the RF until the minimum $\chi^{2}$ is reached. A detailed study of the numerical performance of this procedure as well as the uniqueness of the retrieved atmosphere under different levels of noise in the observations is presented by Borrero et al. (2003a).

The radiative transfer is always performed in the optical depth scale, but the flux tube and its force balance with its surroundings are more naturally described in the geometrical depth scale. The correct functioning of this interplay requires a sufficiently complex procedure. First, a geometrical height scale is assigned to the surrounding atmosphere following the strategy outlined in Gray (1992) and integrating the hydrostatic equilibrium equation (assuming a force free situation $\nabla \times \boldsymbol{B} \| \boldsymbol{B})$,

$\frac{\partial P_{\mathrm{g}, \mathrm{s}}}{\partial \tau_{\mathrm{s}}}=\frac{g}{\kappa_{\mathrm{c}, \mathrm{s}}}$

where $g$ is the solar surface gravitational acceleration and $\tau$ is the optical depth computed using the continuum opacity, $\kappa_{\mathrm{c}}$, at $5000 \AA$. This requires an estimate of the gas pressure at the top of the tabulated atmosphere: $P_{\mathrm{g}, \mathrm{s}}\left(\tau_{\mathrm{s}, \max }\right)$. With this, the gas pressure stratification $P_{\mathrm{g}, \mathrm{s}}\left(\tau_{\mathrm{s}}\right)$ is obtained in the surrounding atmosphere, and since the temperature is obtained from the inversion, the equation of state (ideal gas law including a variable mean molecular weight to account for the ionization of the different species) provides the density: $\rho_{\mathrm{s}}\left(\tau_{\mathrm{s}}\right)$. The relation $\mathrm{d} \tau_{\mathrm{s}}=-\rho_{\mathrm{s}} \kappa_{\mathrm{c}, \mathrm{s}} \mathrm{d} z$ is now integrated setting $z=0$ at $\log \tau_{5}=0$ and thus defining the geometrical height scale.
The gas pressure in the tube component is obtained under the assumption of total pressure balance with the surroundings at the height of the axis of the flux tube,

$P_{\mathrm{g}, \mathrm{t}}(z)= \begin{cases}P_{\mathrm{g}, \mathrm{s}}(z)+\frac{\left(B_{\mathrm{s}}^{2}-B_{\mathrm{t}}^{2}\right)}{8 \pi} & \text { if } z \in\left[z_{0}-R_{\mathrm{t}}, z_{0}+R_{\mathrm{t}}\right] \\ P_{\mathrm{g}, \mathrm{s}}(z) & \text { otherwise. }\end{cases}$

This assumption is valid as long as the magnetic field of the external atmosphere does not penetrate into the flux tube and vice versa (Kippenhahn \& Möllenhof 1975, Chap. 3). Since $P_{\mathrm{g}, \mathrm{t}}(z)$ is now known and $T_{\mathrm{t}}(z)$ was obtained from the inversion, the density $\rho_{\mathrm{t}}(z)$ can be evaluated by using the idal gas equation, and thus a new optical depth scale for the atmosphere containing the flux tube can be obtained through the relation: $\mathrm{d} \tau_{\mathrm{t}}=$ $-\rho_{\mathrm{t}} \kappa_{\mathrm{c}, \mathrm{t}} \mathrm{d} z$. For the integration of this last equation a boundary condition is employed which implies that for $z>z_{0}+R_{\mathrm{t}}$ the surrounding and flux tube components must have the same $z$ values: $z\left(\tau_{\mathrm{t}}\right)=z\left(\tau_{\mathrm{s}}\right)$. Note that the obtained flux tube density, $\rho_{\mathrm{t}}(z)$, does not satisfy vertical hydrostatic equilibrium.

\section{Observations and data reduction}

The active region NOAA AR 8706 was observed on Sep. 21st, 1999 and Sep. 27th, 1999 at $\mu=\cos \theta=0.51$ and $\mu=0.91$ respectively, using TIP (Tenerife Infrared Polarimeter, Martínez Pillet et al. 1999) attached to the spectrograph of the $70 \mathrm{~cm}$ German VTT of the Observatorio del Teide. Here, $\theta$ is the heliocentric angle. The recorded spectral region contains the full polarization profiles of the pair of Fe I lines $15648.5 \AA$ $(g=3)$ and Fe I $15652.8 \AA\left(g_{\text {eff }}=1.53\right)$. The wavelength sampling is about $29 \mathrm{~m} \AA$. The diagnostic properties of these lines have been discussed by Solanki et al. (1992). They are formed in the deep photosphere as a result of their high excitation potentials and the low continuum opacity at this wavelength. These lines sample a relatively narrow layer not wider than $\log \tau_{5}=[0.5,-2]$ (see Bellot Rubio et al. 2000; Mathew et al. 2003). In the umbra the second neutral iron line is heavily blended by two molecular $\mathrm{OH}$ lines at 15651.9 and $15653.5 \AA$. These lines are Zeeman sensitive and belong to the Meinel system 3, 1 (see Berdyugina \& Solanki 2002, for details). Their equivalent widths greatly decrease towards the penumbra. However they can still be seen clearly at the umbrapenumbra boundary. In order to accurately analyze both atomic lines we also compute the $\mathrm{OH}$ lines. The amployed atomic parameters for the observed $\mathrm{Fe}$ I lines were taken from Borrero et al. (2003b), while for the $\mathrm{OH}$ lines the values given by Abrams et al. (1994) and Mies (1974) were used (see Table 1).

The usual data reduction procedures for TIP were in general followed; we proceeded carefully at several points, however. Firstly the neutral iron line, Fe I 15648.5, appears to be blended by a telluric line in its red wing. This complication was overcome by fitting the average quiet Sun profile of the second line with an appropriate model atmosphere. This model was then used to synthetize the first iron line. The ratio between the average observed and computed line allowed us to recover the shape of the telluric blend, which was subsequently eliminated from the rest of the profiles. This procedure, although not perfect, does not introduce modifications to the original profile (equivalent width and line core intensity) larger than $\sim 3 \%$. 
Table 1. Atomic and molecular parameters of the observed spectral lines. $\lambda_{0}$ represents the laboratory central wavelength, $\chi_{1}$ the excitation potential of the lower energy level, and $\log g f$ the logarithm of the oscillator strength times the multiplicity of the level. The parameters $\alpha$ and $\sigma$ (in units of Bohr's radius, $a_{0}$ ) are used to calculate the broadening of the lines by collisions with neutral hydrogen atoms as resulting from the ABO theory (Barklem \& O'Mara 1997). The last column gives the effective Lande factor of the transition, $g_{\text {eff }}$. For the molecular lines $I_{\mathrm{U}}, I_{\mathrm{L}}, V_{\mathrm{U}}$ and $V_{\mathrm{L}}$ represent the upper/low multiplets sublevels and vibrational levels respectively. $J_{\mathrm{L}}$ stands for the rotational number of the lower level. Finally, the oscillator strength of the molecular transition is given.

\begin{tabular}{lcccccc}
\hline \hline Atom & $\begin{array}{c}\lambda_{0} \\
(\AA)\end{array}$ & $\begin{array}{c}\chi_{l} \\
(\mathrm{eV})\end{array}$ & $\begin{array}{c}\log g f \\
(\mathrm{dex})\end{array}$ & & $\begin{array}{c}\sigma \\
\left(a_{0}^{2}\right)\end{array}$ & $g_{\text {eff }}$ \\
\hline \hline Fe I & 15648.515 & 5.426 & -0.675 & 0.229 & 977 & 3.00 \\
Fe I & 15652.874 & 6.246 & -0.043 & 0.330 & 1444 & 1.53 \\
\hline Molecule & $\lambda_{0}$ & Branch & $I_{\mathrm{U}}-I_{\mathrm{L}}$ & $V_{\mathrm{U}}-V_{\mathrm{L}}$ & $J_{\mathrm{L}}$ & $f$ \\
\hline $\mathrm{OH}$ & 15651.895 & $\mathrm{P}$ & $1-1$ & $3-1$ & 6.5 & $0.8 \times 10^{-6}$ \\
$\mathrm{OH}$ & 15653.478 & $\mathrm{P}$ & $1-1$ & $3-1$ & 6.5 & $0.8 \times 10^{-6}$ \\
\hline
\end{tabular}

Secondly, the continuum correction posed a considerable problem. The four quadrant configuration of the TIP camera produce small gradients in the continuum intensity that remain after applying the flat field correction. To account for this we compared our spatially averaged flat field with the infrared FTS atlas from Livingston \& Wallace (1991) and defined several wavelength positions where the continuum should be reached. A smooth second order polynomial is interpolated over those selected points and used to bring the continuum in the flat field image to the same level as the FTS continuum. Thirdly, the polarization signals were corrected for residual cross talk using the statistical approach described in Collados (2001; see also Schlichenmaier \& Collados 2002). Finally, the wavelength was calibrated by assigning the laboratory wavelengths to the cores of the Fe I lines in the average quiet Sun profiles. To account for the effects of the granulation we shifted this wavelength scale by $-400 \mathrm{~m} \mathrm{~s}^{-1}$, which is the approximate value for the convective blueshift as deduced from the quiet sun model of Borrero \& Bellot Rubio (2002). Second order corrections due to the different viewing angles (Balthasar 1985) have not been performed.

The seeing conditions were rather good during the observations, with the granulation being clearly discernible in the reconstructed continuum images (see Fig. 2; left panels). By calculating the power spectrum of the continuum intensity in the neighbouring quiet Sun we estimate the spatial resolution to be about 1 arcsec. In Fig. 2 (right panels) the maps of the total circular polarization are also shown. Due to projection effects the polarity inversion line (region in the limb side of the penumbra where the Stokes $V$ signal changes its sign, i.e., where the average magnetic field is perpendicular to the observer) appears in the outer penumbra when the sunspot is observed near disk center $(\mu=0.91)$ but lies closer to the umbra when the spot is located closer to the limb $(\mu=0.51)$. Note that the arrows in Fig. 2 point towards the solar disk center. Part of this data set has been analyzed in Paper I (see also Mathew et al. 2003, 2004).

\section{Results and discussion}

We have inverted individually all the pixels along the radial cuts shown in Fig. 2 (5 for each heliocentric angle). Each cut contains approximatively 20 pixels and ranges roughly from $r / R=0.4$ to $r / R=0.9$ (where $R$ is the penumbral radius, indicated by the external contour in Fig. 2). We have chosen the cuts such that they all lie on the limbward side of the penumbra and near the line of symmetry (i.e. the line connecting the sunspot's center and the center of the solar disk), since this is where the flux tubes leave the most distinctive fingerprints on the observed profiles, thus allowing for a reliable determination of their properties, as shown in Paper I.

\subsection{Example}

In Fig. 3 we present an example of the observed (filled circles) and fitted (solid lines) circular polarization profiles for a penumbral point. These multi-lobed profiles can be successfully fitted by adding together two Stokes $V$ profiles. The profile from the surrounding atmopshere, $V_{\mathrm{s}}$, is produced by a positive polarity magnetic field $\left(\gamma<90^{\circ}\right.$; dot-dashed line) with zero line-of-sight velocity (zero crossing is at $\lambda_{0}$ ), and the Stokes $V$ profile from the ray cutting through the flux tube, $V_{\mathrm{t}}$, which is produced by a negative polarity magnetic field $\left(\gamma>90^{\circ}\right.$; dashed line) carrying a flow directed away from the observer (zero crossing is red shifted with respect to $\lambda_{0}$ ). The filling factor of the flux tube atmosphere $\alpha_{\mathrm{t}}$ and of the stray light contribution $\alpha_{\mathrm{q}}$ are applied to $V_{\mathrm{s}}$ and $V_{\mathrm{t}}$ to obtain the final emergent profile (solid line) according to Eq. (2).

In Fig. 4 we present the atmospheric stratifications resulting from the inversion of the profiles shown in Fig. 3 using the uncombed model (see Sect. 2). Outside the flux tube the magnetic field strength is about 2000 Gauss and is inclined with respect to the observer by about $75^{\circ}$. Inside the flux tube the magnetic field is weaker $(\simeq 1300$ Gauss) and more inclined $\left(\gamma \simeq 100^{\circ}\right)$. While the surrounding atmosphere is basically at rest, $V_{\mathrm{LOS}, \mathrm{s}} \simeq 0.2 \mathrm{~km} \mathrm{~s}^{-1}$, we detect red shifts of $V_{\mathrm{LOS}, \mathrm{t}} \simeq 1.1 \mathrm{~km} \mathrm{~s}^{-1}$ inside the flux tube. In addition, the results from the inversion indicate that the flux tube is hotter, by roughly $500 \mathrm{~K}$, than the surrounding atmosphere. The presence of the discontinuity along the flux tube atmosphere produces the area asymmetry in Stokes $V, \delta A_{\mathrm{FIT}}$, marked in Fig. 3.

\subsection{General properties}

We have taken the individual results of the inversions of the considered radial cuts (separately for each heliocentric angle) at the geometrical height $z=z_{0}$ (i.e. at the location of the tube axis), and calculated the averages at each radial position in the sunspot. This is done individually for the flux tube component and the surrounding magnetic field. The results are presented in Figs. 5 and 6 for the temperature, line-of-sight velocity, the flux tube's filling factor $\alpha_{\mathrm{t}}$, magnetic field zenith angle and magnetic field strength. In order to compare the magnetic field 

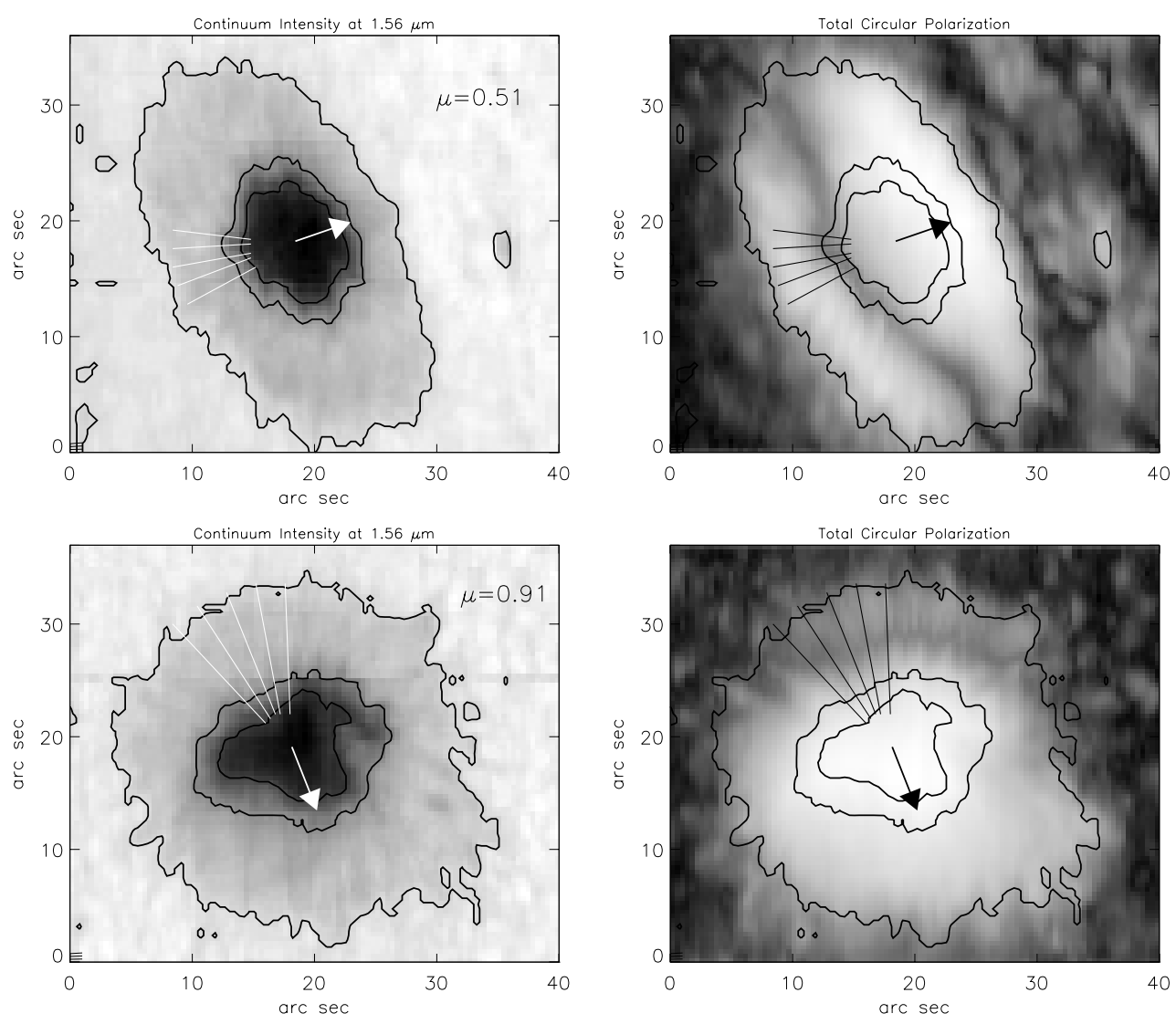

Fig. 2. Top panels: NOAA 8706 observed in 21 September 1999 at a heliocentric angle $\mu=0.51$ (left: continuum intensity map at $1.56 \mu$ m; right: total circular polarization map for Fe I $15648.5 \AA$ A). Bottom panels: NOAA 8706 observed in 27 September 1999 at a heliocentric angle $\mu=0.91$ (left: continuum intensity map at $1.56 \mu \mathrm{m}$; right: total circular polarization map for Fe I $15648.5 \AA$ ). The arrows point towards the direction of the solar disk center. The two inner-most contours enclose the umbral-penumbral boundary. The external contour defines the sunspot radius $r=R$, at each position angle. These three contours have been defined as $0.45 I_{\mathrm{c}}, 0.65 I_{\mathrm{c}}$ and $0.85 I_{\mathrm{c}}$, where $I_{\mathrm{c}}$ represents the average continuum intensity of the quiet Sun. The radial cuts selected for our analysis are also shown.
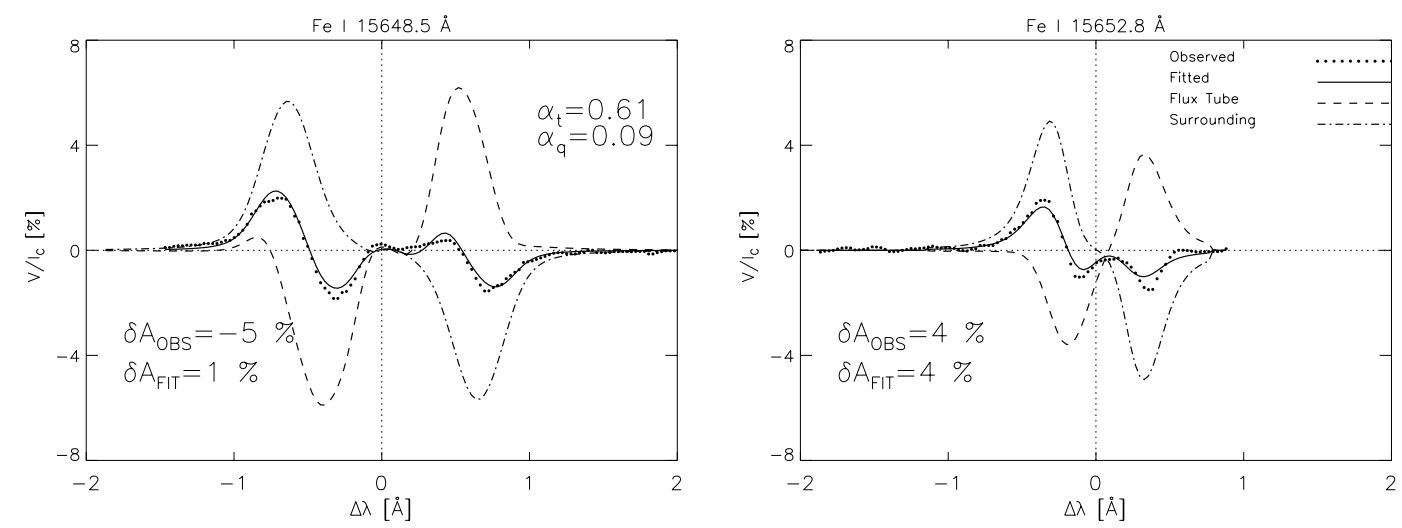

Fig. 3. Example of the observed (dots) and fitted (solid lines) Stokes $V$ profiles (left: $15648.5 \AA$; right: $15652.5 \AA$ ) for a penumbral point. The fitted profile is obtained by linearly combining the profile emerging from the surrounding component (dot-dashed line) and the profile emerging from the ray piercing the flux tube (dashed line). The employed filling factors, $\alpha_{\mathrm{t}}$ and $\alpha_{\mathrm{q}}$, as well as the area asymmetry of the observed and fitted profiles are also given.

inclination deduced for sunspots located at different heliocentric positions we have converted from the observer's reference frame, $\gamma$, to the local reference frame. Therefore, we plot now the zenith angle, $\zeta$ (Fig. 6; top panels). A zenith angle smaller than, equal to or larger than $90^{\circ}$ indicates that the magnetic field is inclined upwards, lies parallel to or is inclined downwards with respect to the solar surface. All in all, the results for the two sets of observations are remarkably similar in spite of the different viewing angles and the time difference of 6 days between the two observations. 

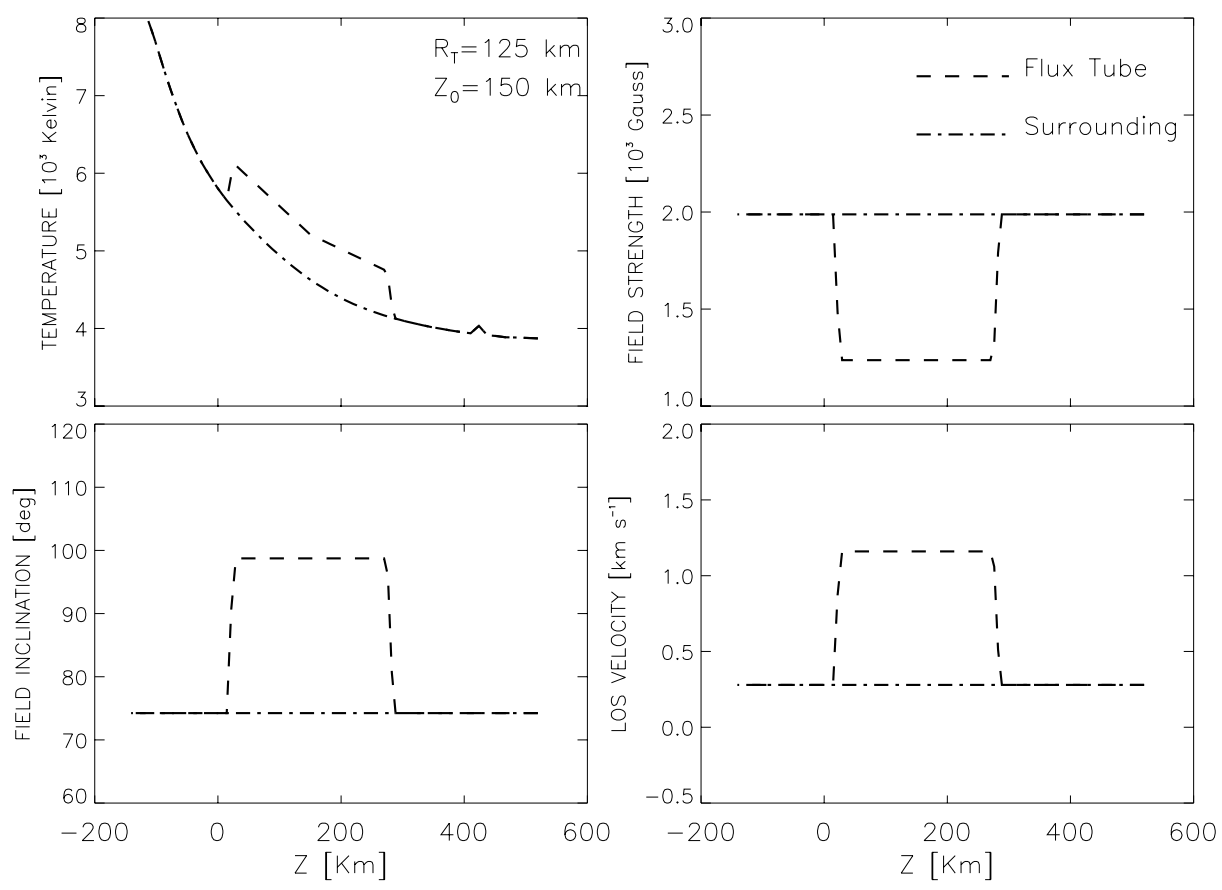

Fig. 4. Temperature (top-left panel), magnetic field strength (top-right panel), magnetic field inclination (bottom-left) and line-of-sight velocity (bottom-right) for the flux tube atmosphere (dashed lines) and its surroundings (dot-dashed lines) as a function of the geometrical depth, obtained from the inversion of the profiles in Fig. 3. The flux tube's radius returned by the inversion is $125 \mathrm{~km}$ and its central position is $z_{0}=150 \mathrm{~km}$.

In the inner penumbra, we detect nearly, but not completely horizontal flux tubes $\left(\zeta_{\mathrm{t}} \simeq 70-80^{\circ}\right)$ that are hotter than their surroundings by about $500-1000 \mathrm{~K}$. These flux tubes carry most of the Evershed flow, with LOS velocities in the inner penumbra ranging from 0.5 to $3 \mathrm{~km} \mathrm{~s}^{-1}$. The magnetic field strength in the flux tubes is around 1500 Gauss. The atmosphere surrounding these flux tubes possesses a more vertical $\left(\zeta_{\mathrm{s}} \simeq 20-40^{\circ}\right)$ and stronger magnetic field $(B \simeq 2300-2500)$. No signatures of the Evershed flow are detected here.

As the radial distance increases, the flux tubes cool down to temperatures similar to those of the surroundings. At large radial distances $r / R \geq 0.7$ the temperature in the flux tube component decreases even below the surrounding temperature, although only slightly $(\simeq 200-300 \mathrm{~K})$. At the same time the tubes become more horizontal, reaching $\zeta_{\mathrm{t}} \simeq 90^{\circ}$, and point slightly downwards with respect to the solar surface, $\zeta_{\mathrm{t}} \simeq 95-100^{\circ}$, near the outer edge of the penumbra, i.e. at $r / R \geq 0.8$. In addition, their magnetic field strength decreases slowly to $800-1000$ Gauss at $r / R=0.9$ while the LOS velocity increases monotonically in both spots, although for $\mu=0.51$ it suffers a sudden drop near the outer penumbral boundary. The filling factor of the flux tubes (Fig. 5; bottom panels) increases continuously from very small values at the inner penumbra $\alpha_{\mathrm{t}} \simeq 0.2-0.3$ until they cover almost all the resolution element at the outer boundary $\alpha_{\mathrm{t}} \simeq 0$.9. This can be interpreted either as an increase in the horizontal cross section of the flux tubes or as an increase in the number of flux tubes per resolution element.

The surrounding atmosphere exhibits a quite different behaviour. The magnetic field strength decreases very rapidly towards the outer penumbra, reaching similar values to those of the flux tube's magnetic field strength $(\simeq 800-1000$ Gauss $)$ at $r / R \gtrsim 0.8$. The inclination of the magnetic field increases slightly with radial distance up to $\zeta_{\mathrm{s}} \simeq 40-50^{\circ}$. The LOS velocities remain small throughout the penumbra.

Before discussing the individual results in more detail there are several points which need to be clarified. First of all, we want to stress that the results obtained from the inversions of the radial cuts when the spot is near the disk center (Figs. 5 and 6; left panels) are more reliable at intermediate to large radial distances, while results inferred from the sunspot at large heliocentric angles (Figs. 5 and 6; right panels) are more reliable in the inner penumbra. The reason for this is that the magnetic neutral line is located in the outer penumbral for sunspots near the disk center, $\mu=0.91$, but it shifts towards the umbra as the sunspot is located closer to the limb $(\mu=0.51)$. The magnetic neutral line is where multi-lobed Stokes $V$ profiles are commonly observed (Sánchez Almeida \& Lites 1992; Schlichenmaier \& Collados 2002). As already discussed in Paper I inferred parameters from the inversion are more reliable for exactly such complex profiles, since the signature of the two unresolved components (flux tubes and magnetic surrounding) can be better distinguished there (e.g. Fig. 3), allowing for a more reliable inference of their properties. In general, however, larger and more accurate line-of-sight velocities are obtained from the sunspot near the limb (at all radial distances) since the Evershed flow is more aligned with the line-of-sight there.

We note that the possible size of the flux tubes can be much smaller than our spatial resolution of 1 arcsec (Sütterlin 2001; Sütterlin et al. 2004; Scharmer et al. 2002; Rouppe van der Voort et al. 2004). Threfore the deduced properties are unlikely to correspond to one single flux tube but 

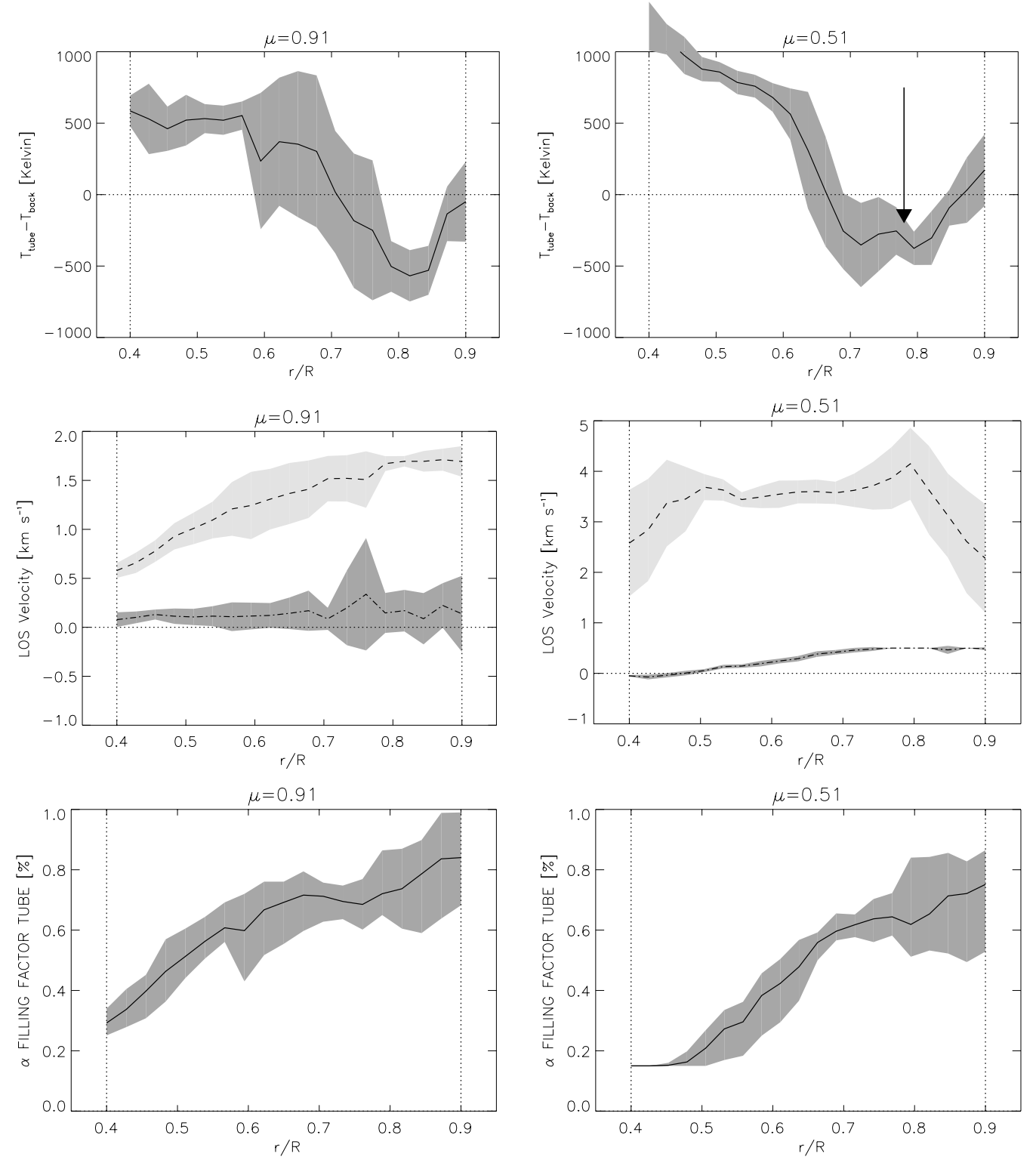

Fig. 5. Top panels: temperature difference between the flux tube atmosphere and its surroundings as a function of radial distance from the spot center. Middle panels: radial variation of the line-of-sight velocities inside (dashed lines) and outside the flux tubes (dashed-dotted lines). Bottom panels: radial variation of the flux tube's filling factor, $\alpha_{\mathrm{t}}$. Left panels: sunspot at $\mu=0.91$. Right Panels: sunspot at $\mu=0.51$. All quantities refer to the central position of the tube, $z=z_{0}$. Shaded areas denote the maximum deviations from the average at each radial position, obtained from the individual inversions of all the radial cuts. The arrow indicates the approximate radial position where the flux tube experiences a final temperature enhancement (see Sect. 6.4).

rather to some average over all the possible penumbral fibrils contained in the resolution element. However, as one can see from Figs. 5 and 6, the inferred properties are similar to those expected for a single flux tube which crosses the penumbra from its inner to the outer boundary.

\section{Width of the penumbral filaments and Stokes $V$ area asymmetry}

The area asymmetry, $\delta A$, of the circular polarization is defined as:

$\delta A=\frac{\int V(\lambda) \mathrm{d} \lambda}{\int|V(\lambda)| \mathrm{d} \lambda}$
It is different from zero whenever gradients along the line-ofsight of the magnetic field vector and LOS velocity are present (Landolfi \& Landi degl'Innocenti 1996). Solanki \& Montavon (1993) realized that the huge gradients needed to reproduce the area asymmetry observed in the sunspot penumbra with the visible Fe I lines at 6301.5 and $6302.5 \AA$ (Sánchez Almeida \& Lites 1992) could be interpreted as a horizonal flux tube embedded in a more vertical background. As the line of sight crosses the tube's boundaries the physical stratifications describing the atmosphere suffer a jump that is directly responsible for the generation of the area asymmetry in Stokes $V$. Schlichenmaier et al. (2002) and Müller et al. (2002) further investigated this issue and pointed out that the area 

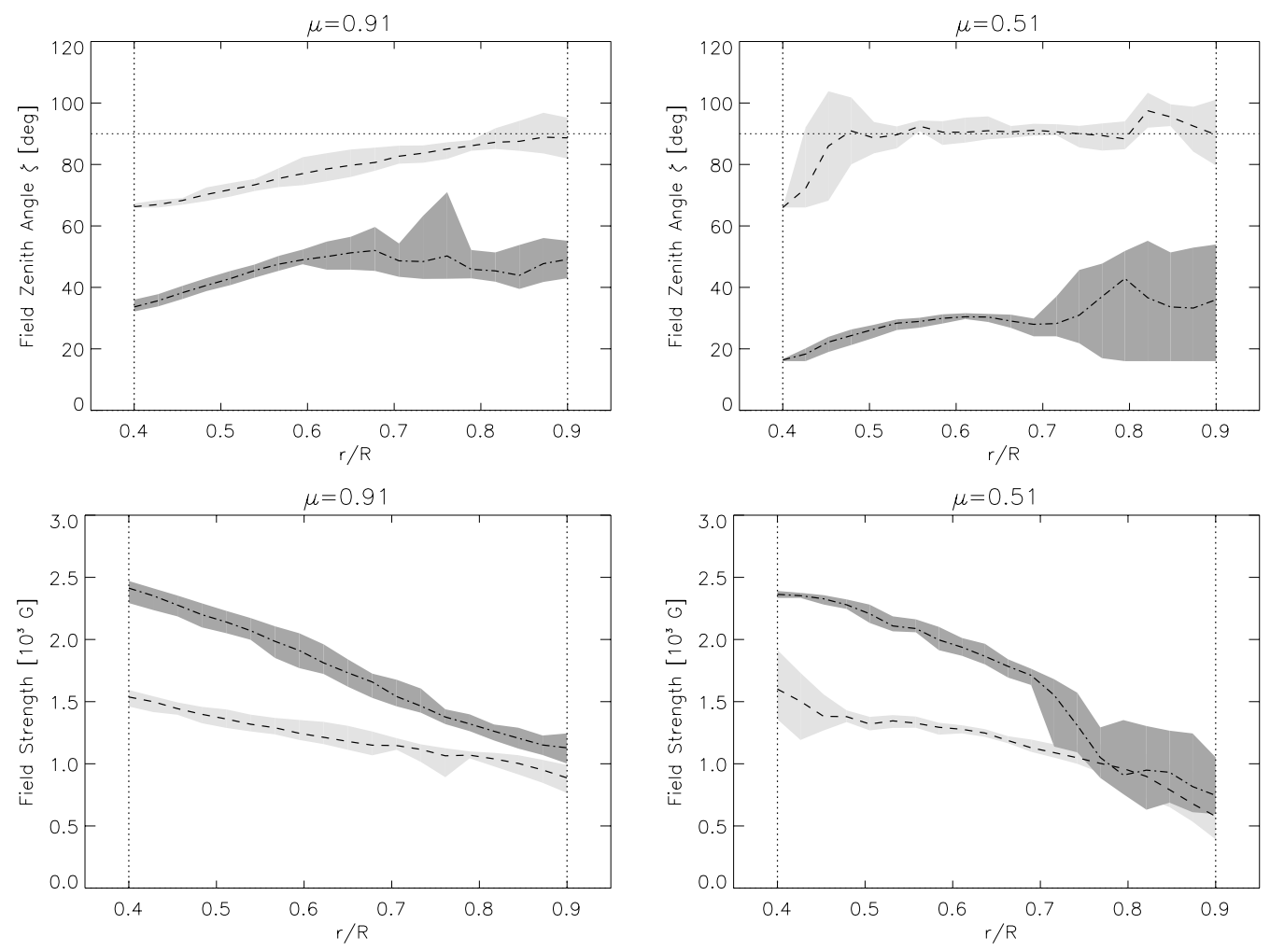

Fig. 6. The same as Fig. 5, but now for the radial variation of the magnetic field zenith angle (top panels) and magnetic field strength (bottom panels).

asymmetry observed in the visible Fe I $6301 \AA$ A lines is dominated by jumps in the magnetic field inclination, while the area asymmetry observed in the infrared $1.56 \mu \mathrm{m}$ lines can be explained in terms of jumps in the magnetic field azimuth. Both cases are compatible with the uncombed penumbra proposed by Solanki \& Montavon (1993).

As already described in Sect. 2, in the uncombed model, the discontinuities needed to produce asymmetric circular polarization profiles are located at the tube's boundaries: $z^{*}=z_{0} \pm R_{\mathrm{t}}$, with $R_{\mathrm{t}}$ and $z_{0}$ (the tube's radius and central position) being free parameters of the inversion. The radius we obtain from the inversion of all the pixels contained in the 10 radial cuts in Fig. 1 in the same sunspot at two different heliocentric angles is, consistently, $125 \mathrm{~km}$, which is precisely the maximum value we allow for $R_{\mathrm{t}}{ }^{3}$. This means that the inversion tries to make the flux tube as thick as possible. The area asymmetry of the synthetic profiles, $\delta A_{\mathrm{FIT}}$ only poorly reproduces the observed one: $\delta A_{\mathrm{OBS}}$ (see Fig. 7; top panels). In most cases the uncombed model produces an area asymmetry which is very little or almost zero, and varies over a smaller range than $\delta A_{\mathrm{OBS}}$.

The small observed area asymmetries (average of $\delta A_{\mathrm{OBS}} \sim$ $3 \%$ ) clearly indicate that $\delta A$ is a parameter which plays a minor role in the inversion since the shape of the circular polarization profiles can be successfully fitted by means of profiles showing little or even no area asymmetry at all (as the $2 \mathrm{C}$ model

3 This maximum value is set in the inversion code so that the upper boundary of the flux tube lies below the top of the tabulated atmosphere. This ensures that the condition $z\left(\tau_{\mathrm{t}}\right)=z\left(\tau_{\mathrm{s}}\right)$ for $z>z_{0}+R_{\mathrm{t}}$ (see Sect. 2) can be applied. in Paper I). Even extremely strange profiles, such as those presented in Fig. 3, can be fitted reasonably well. This is because the circular polarization profiles of the spectral lines used in this work are mainly affected by the presence of two different polarities in the resolution element (background with $\gamma<90^{\circ}$ and flux tube with $\gamma>90^{\circ}$ ) rather than by discontinuities along the line of sight in the physical parameters. This is due to the large Zeeman splitting of these loines and the fact that they are not strongly saturated (see Grossmann-Doerth et al. 1989). Note that Borrero et al. (2004; Paper I) also reached the same conclusion and suggested that using the visible iron lines at Fe I $6301 \AA$, whose area asymmetry is far more sensitive to such discontinuities, might help to further constrain the size of the penumbral filaments. For the case of the infrared Fe I lines at $1.56 \mu \mathrm{m}$ they constrained a small region where the area asymmetry of these lines is sensitive to gradients along the line of sight: $\log \tau_{5} \in[0,-0.5]$.

In Fig. 7 (middle panels) the position (in the optical depth scale) of the tube's lower boundary is plotted as a function of $r / R$. At $r / R<0.7$ the lower boundary is located at $\log \tau_{5}>$ 0 . At the outer penumbra, however, the lower boundary shifts to higher layers: $\log \tau_{5} \sim-0.25$, where the discontinuity is effective in generating area asymmetry (see Paper I). Note that for most of the penumbra the lower boundary also remains below the $\log \tau_{1.56}$ level (dashed line). For comparison we plot in Fig. 7 (bottom panel) the observed, $\delta A_{\mathrm{OBS}}$ (solid line), and fitted (dashed line), $\delta A_{\mathrm{FIT}}$, area asymmetry for all the considered radial cuts versus $r / R$. $\delta A_{\mathrm{OBS}}$ increases radially in the penumbra, from small negative values $(\sim-2 \%)$ up to larger positive ones: $\sim 7 \%$. Interestingly the fitted area asymmetry displays a 

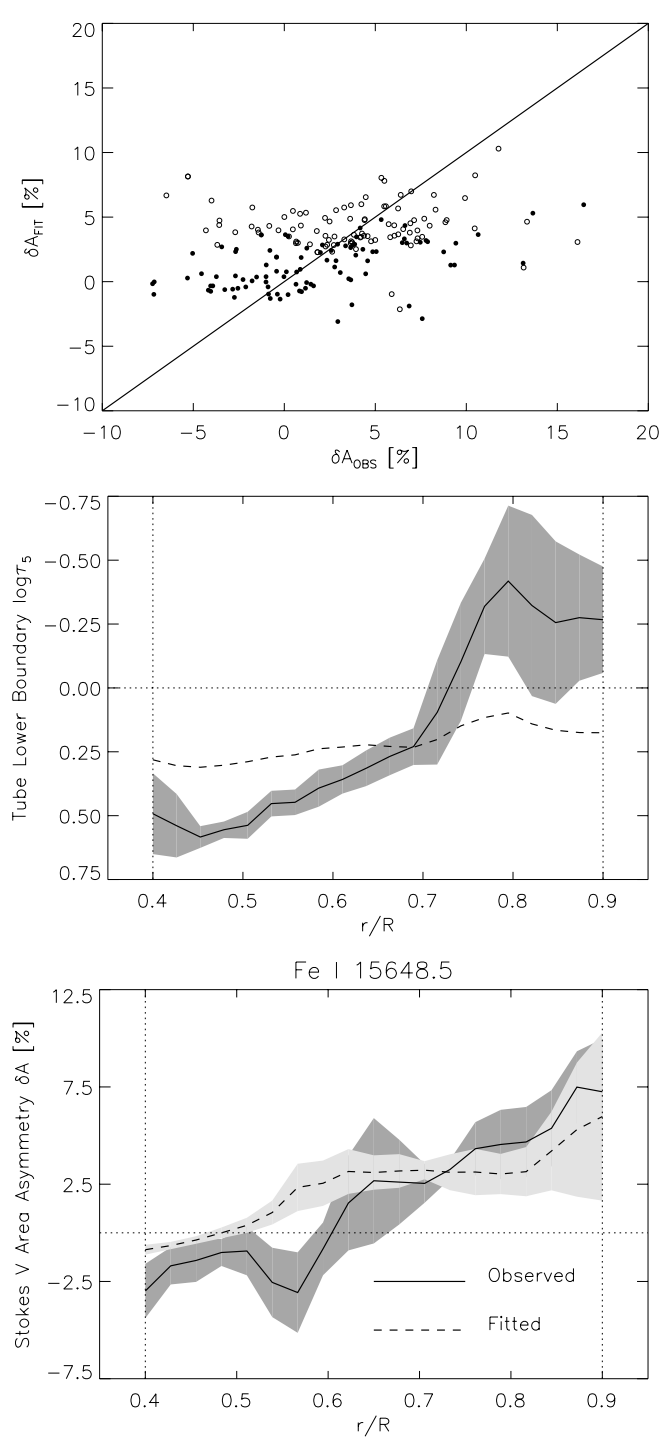

Fig. 7. Top panel: area asymmetry from the best-fit Stokes $V$ profiles, $\delta A_{\mathrm{FIT}}$, versus area asymmetry of the observed Stokes $V, \delta A_{\mathrm{OBS}}$, for both Fe I lines for the case $\mu=0.91$. For $\mu=0.51$ (not shown) the result is very similar. Filled circles are for Fe I $15648.5 \AA$, open circles for $15652.8 \AA$ A. Middle panel: average position, in the optical depth scale, of the lower boundary of the flux tube (solid line). The $\log \tau_{1.56}=0$ level is indicated by the dashed line. Maximum and minimum individual deviations of the 10 radial cuts considered are indicated by the shaded area. Bottom panel: radial variation of the fitted (dashed line) and observed area asymmetry (solid line) of Fe I $15648.5 \AA$.

similar behaviour, specially in the outer penumbra, where observed and fitted values become similar.

The combination of these results leads us to conclude that, in the inner-intermediate penumbra, where the observed area asymmetry in the circular polarization profiles of the Fe I $1.56 \mu \mathrm{m}$ lines is small, discontinuities are not important to reproduce the profiles and therefore the tube radius is a parameter which is not well constrained from the inversion. However, in the outer penumbra, the observed area asymmetry becomes large enough to turn into an important ingredient to successfully fit the circular polarization profiles. In these regions, the lower boundary of the flux tube is located at heights where it is effective in generating area asymmetry. As the upper boundary remains unseen we still cannot draw any reliable conclusion on the actual size of the penumbral filaments.

Given that Fe I lines at $1.56 \mu \mathrm{m}$ do not see much of the tube's boundaries, our uncombed model could in principle be simplified into a two component model where all physical quantities are constant with height (e.g., making the tube's radius infinite in Fig. 4). Such models have been previously used to study the fine structure of the penumbra (Bellot Rubio et al. 2003, 2004; Borrero et al. 2004). However a feature included in the uncombed model but has been neglected by previous two component models remains: the use of the total pressure balance between the flux tube and its surroundings. As we shall discuss in Sect. 6.1, this has important consequences.

\section{Nature of the Evershed flow}

Previous observational analyses, which neglected the fact that in our resolution element we have mixed signals coming from the flux tubes and their magnetic surroundings, obtained that the magnetic field strength showed a strong radial decrease from $B_{\text {inner }} \simeq 2500 \mathrm{G}$ to $B_{\text {outer }} \simeq 1000 \mathrm{G}$ (Beckers \& Schröter 1969; Wittmann 1974; Lites \& Skumanich 1990; McPherson et al. 1992; Lites et al. 1993; Keppens \& Martínez Pillet 1996; Stanchfield et al. 1997). This implies that the magnetic field is larger at the inner footpoint of a siphon flow carrying loop than in the outer one, making it difficult for this mechanism to work.

If the fine structure of the penumbra is taken into account (Rüedi et al. 1998, 1999; Borrero et al. 2004) a more favourable situation appears: an inclined magnetic field whose strength rapidly decreases with radial distance and an almost horizontal magnetic field carrying the Evershed flow with a far smaller radial drop in field strength are inferred (see Fig. 6; bottom panels). The small decrease in the field strength for the component carrying the Evershed flow still implies a stronger magnetic field at small $r / R$, but now the difference between inner and outer penumbra is significantly reduced to $\simeq 300-500 \mathrm{G}$. Although this finding on its own is insufficient to produce an outwards accelerated flow it represents a more favourable situation for the siphon flow mechanism, specially if we take into account that, as noticed by Montesinos \& Thomas (1997), the inner and outer footpoints, as measured by observations, are not necessarily at exactly the same height.

\subsection{Gas pressure gradient}

According to Fig. 6 (bottom panels) the magnetic field strength of the flux tubes is much smaller than that of the surrounding atmosphere in the inner penumbra at a geometrical height $z=z_{0}$ (central position of the tube). By requiring total pressure balance between the flux tube interior and the external atmosphere (Eq. (4)) one can easily deduce that $P_{\text {gas, } t}\left(z_{0}\right) \gg P_{\text {gas }, \mathrm{s}}\left(z_{0}\right)$. At large radial distances the situation is such that the magnetic field strength in the flux tube and its surrounding atmosphere becomes very similar and therefore: $P_{\text {gas }, \mathrm{t}}\left(z_{0}\right) \simeq P_{\text {gas, }}\left(z_{0}\right)$. In Fig. 8 we plot the actual $\Delta P_{\text {gas }}=P_{\text {gas }, \mathrm{t}}\left(z_{0}\right)-P_{\text {gas }, \mathrm{s}}\left(z_{0}\right)$ as a function of radial distance in the penumbra (top panel for $\mu=0.91$ 

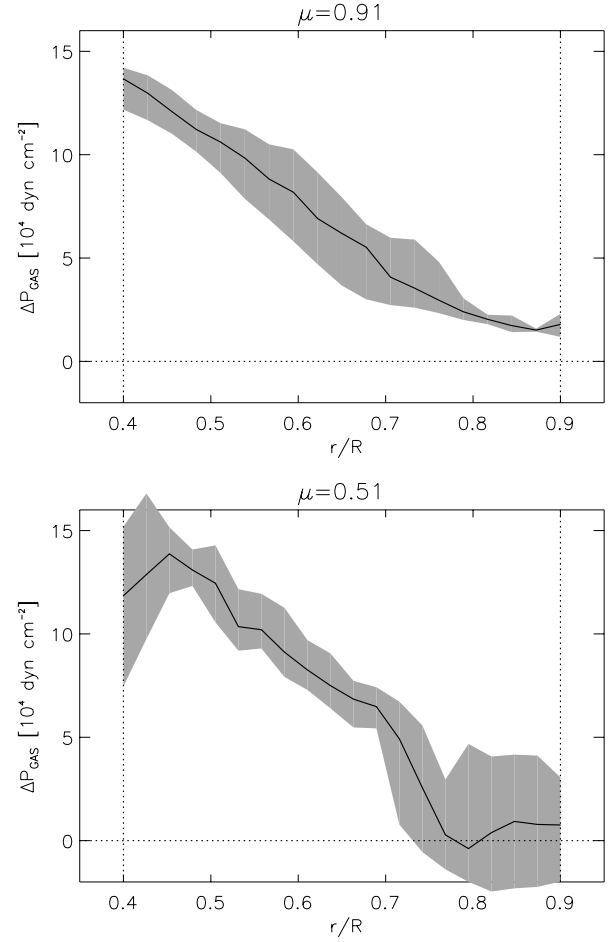

Fig. 8. Radial variation of the gas pressure difference, $\Delta P_{\text {gas }}$, between the flux tube and its surrounding atmosphere at $z=z_{0}$. Top panel: for NOAA 8706 at an heliocentric angle of $\mu=0.91$. Bottom panel: the same for $\mu=0.51$.

and bottom panel for $\mu=0.51$ ). As can be seen that this difference decreases almost linearly with $r / R$, reaching values close to zero near the outer penumbral boundary.

Unfortunately, this effect does not imply a radial decrease in the gas pressure inside the flux tube, because of the unknown radial behaviour of the external pressure $P_{\text {gas,s }}\left(z_{0}\right)$. In particular, its calculation is ill-posed by the boundary condition described in Sect. 2, where a value of the gas pressure at the highest photospheric layers is prescribed for the surrounding atmosphere. This is done for all pixels independently of their position on the spot: $P_{\text {gas, } s}\left(\tau_{\max }\right)=P_{0}$. Consequently, the geometrical height scale $z_{\mathrm{s}}\left(\tau_{\mathrm{s}}\right)$ in the surrounding atmosphere does not take into account pixel to pixel variations of the absolute geometrical height scale (i.e. Wilson depression). The radial variation of the difference in gas pressure between the flux tube and the surrounding atmosphere, however, only depends on the difference in the magnetic field strength between them and is calculated under the condition of total pressure balance (magnetohydrostatic constraint). Although it cannot directly prove that there is a radial decrease in the gas pressure along the flux tube axis, it does provide a strong indication that this is indeed the case.

\subsection{Cooling flux tubes}

Our results show that in the inner penumbra the flux tubes are hotter (by roughly $500-1000 \mathrm{~K}$ ) than their surroundings. As we move to larger radial distances the temperature becomes very similar inside and outside the flux tube. This is in very good agreement with the theoretical predictions of the moving flux tube model (Schlichenmaier et al. 1998a,b). The result that at a given point, $r / R \simeq 0.7$, the flux tube becomes cooler than its surroundings can be explained by assuming that the flow becomes adiabatic due to a less efficient radiative exchange in the higher parts of the anchored flux tube (Montesinos \& Thomas 1997). The temperature increase seen at larger radial distances is explained in terms of a shock front (see Sect. 6.3).

This picture indicates that at the umbral-penumbral boundary, a first set of hot flux tubes (small ones or a small number of them according to the tiny filling factor inferred; see Fig. 5, bottom panels), appear. Remarkably, these flux tubes that have just emerged already carry strong velocities (see Fig. 5, middle panels), perhaps as an indication that most part of the plasma acceleration has already occurred. Again this is in very good agreement with the moving flux tube simulations (see Schlichenmaier et al. 1998a,b) where the acceleration takes place in the inner penumbra, where hot flux tubes carrying plasma at about $8000-10000 \mathrm{~K}$ rapidly cool down. The opacity in such extremely hot tubes would be too large to make them observable; therefore what we see is perhaps, the latest stages of this cooling process. Needless to say, hotter flux tubes in the inner penumbra were already expected considering that at small radial distances, the magnetic field strength in the flux tubes is much smaller than in the surrounding atmosphere, and therefore, in order to keep the horizontal total pressure balance between these two atmospheres the gas pressure in the flux tube component has to increase.

\subsection{Shock front}

Another point of special interest is the magnitude of the Evershed flow. Theoretical models predict different values for the speed of the flow inside penumbral flux tubes. Stationary siphon flow models (Thomas \& Montesinos 1991, 1993; Montesinos \& Thomas 1997) distinguish between subcritical and supercritical velocities with respect to the characteristic critical tube speed (Thomas 1988; Ferriz Mas 1988). Transitions between both regimes can be present within the flux tube, leading to shock fronts. Time-dependent simulations of thin flux tubes in the penumbra predict supercritical flows in most of the penumbra regardless of whether the flux tubes remain horizontal beyond the visible limit of the sunspot (Schlichenmaier et al. 1998a,b) or bend back within the penumbra (Schlichenmaier 2002). Taking into account the fine structure of the penumbra, large velocities $\left(>4 \mathrm{~km} \mathrm{~s}^{-1}\right)$ are favoured (Wiehr 1995; del Toro Iniesta et al. 2001; Bellot Rubio et al. 2003, 2004). Most of these values have been obtained under the assumption that the magnetic field and the velocity vectors are mutually parallel (see Bellot Rubio et al. 2003).

The absolute flux tube velocity is: $v_{\mathrm{t}}=v_{\text {los, }} / \cos \gamma_{\mathrm{t}}$, where $\gamma_{\mathrm{t}}$ is the inclination of the tube's magnetic field vector with respect to the observer (both $v_{\text {los, },}$ and $\gamma_{\mathrm{t}}$ are obtained from the inversion). $v_{\mathrm{t}}$ is plotted in Fig. 9 (top left panel) for the sunspot at an heliocentric angle of $\mu=0.51^{4}$. Also plotted are

\footnotetext{
${ }^{4}$ Results for $\mu=0.91$ are not considered, since dividing by the cosine of inclination angles close to $90^{\circ}$ enhances any error in line-ofsight velocities.
} 

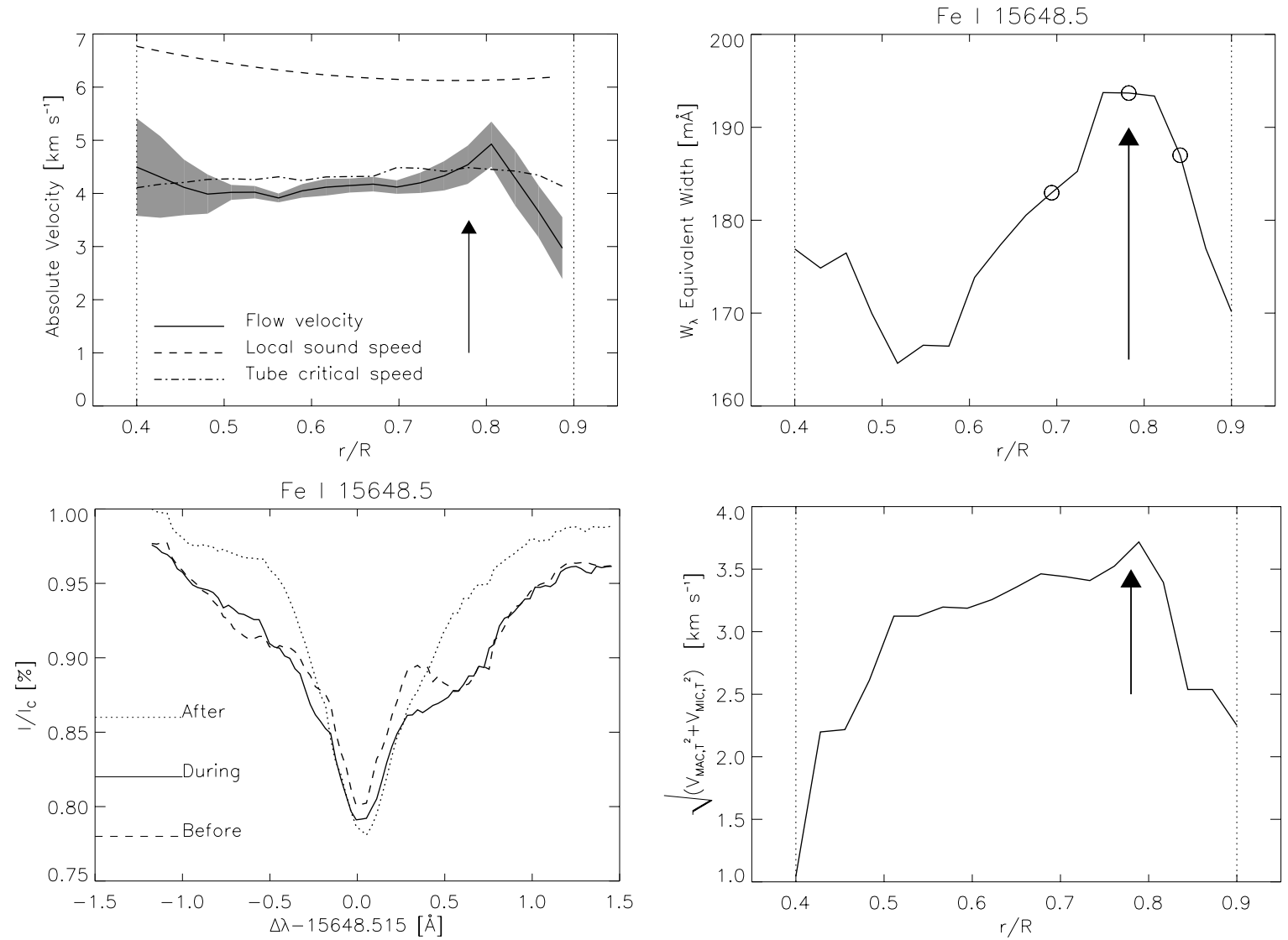

Fig. 9. Top left panel: radial variation of the flow velocity inside the flux tube (solid line). Local sound speed and tube's critical speed are also plotted (dashed and dashed-dotted lines respectively). The vertical arrow marks the position where the flow speed becomes supercritical, $r / R \simeq 0.78$. Top right panel: radial variation of the equivalent width for Fe I $15648.5 \AA$ Aine. The open circles are three selected radial positions before, during and after the shock occurs. Bottom left panels: intensity profiles corresponding to the 3 selected radial positions (top right panel in this figure) before (dashed line), during (solid line) and after the shock (dotted line). Bottom right panels: radial variation of $\sqrt{v_{\text {mic,t }}^{2}+v_{\text {mac, },}^{2}}$.

the local sound speed $c_{\mathrm{S}} \sim T^{1 / 2}$ and the tube's critical speed $c_{\mathrm{t}}=c_{\mathrm{s}} c_{\mathrm{a}} / \sqrt{c_{\mathrm{s}}^{2}+c_{\mathrm{a}}^{2}}$, with $c_{\mathrm{a}} \sim B \rho^{-1 / 2}$ being the Alfvén speed. The velocity in the flux tube always remains subsonic, although we cannot rule out the possibility for supersonic values in the penumbra (del Toro Iniesta et al. 2001; Penn et al. 2003) given the limited number of radial cuts we are considering.

At almost all radial distances, $v_{\mathrm{t}}<c_{\mathrm{t}}$ as well, except for a few regions in the inner $(r / R \simeq 0.4)$, and the outer penumbra $(r / R \simeq 0.78)$. Given the error bars, we do not deem the first case to be reliable. However, for large radial distances (arrow in Fig. 9, top left panel) it seems plausible that the velocity becomes supercritical. Remarkably, after this happens, the velocity suddenly drops again by roughly $2 \mathrm{~km} \mathrm{~s}^{-1}$ to subcritical values at larger distances: $r / R>0.85$. This is accompanied by an increase in the flux tube temperature of about $300-400 \mathrm{~K}$ at these locations (see arrow in Fig. 5; top right panel). Indeed, this is the behaviour expected from a transition between supercritical to subcritical velocities in penumbral flux tubes (Montesinos \& Thomas 1997) produced by a shock front that dissipates kinetic energy and heats the gas. The presence of shocks in the siphon flows has been predicted by numerous authors (Meyer \& Schmidt 1968; Degenhardt 1989, 1991; Thomas \& Montesinos 1991, 1993; Montesinos \& Thomas 1997; Schlichenmaier et al. 1998a,b;
Schlichenmaier 2002), but this is the first time that direct observational evidence of such a shock front within the penumbra is provided.

Shock fronts, if present, are likely to produce an enhancement both in the line width (e.g. FWHM) and in the equivalent width (see Degenhardt et al. 1993; Solanki et al. 1996). In the penumbra, the radial variation of the line width is dominated by the Zeeman splitting (magnetic field). However, the magnetic field affects the equivalent widths of the comparatively weak (i.e. unsaturated) lines considered here only very slightly. In Fig. 9 (top right panels) we plot the radial variation of the equivalent width for Fe I 15648.5 A. It clearly shows an enhancement $(\simeq 10 \%)$ at roughly the same radial distance where the flow speed becomes supercritical (see vertical arrow). Three different radial positions, corresponding to locations before, during and after the shock have been marked with open circles. Intensity profiles for Fe I $15648.5 \AA$ at those locations have been extracted and plotted together in Fig. 9 (bottom left panel). The intensity profile before the shock (dashed line) shows an enhancement in the red wing which is produced by the strongly red shifted flux tube contribution (i.e. satellite line; see Stellmacher \& Wiehr 1980; Wiehr et al. 1986; Wiehr 1995, 1997). During the shock (solid line) the equivalent width of the redshifted component is enhanced. This 
broadening is likely to be produced by a new structure in our resolution element that our model does not account for. Therefore, the only way for the inversion code to make the profile broader (within the constraints of the chosen model) is to increase the micro and macroturbulent velocities. In Fig. 9 (bottom right panel) the line broadening velocity in the flux tube, defined as: $v_{\text {broad }}=\sqrt{v_{\text {mic,t }}^{2}+v_{\text {mac,t }}^{2}}$ is plotted. It shows a peak exactly at the radial positions at which the flow speed becomes supercritical. This provides a strong indication that this spectral signature really corresponds to a shock front in the flux tube at large radial distances in the penumbra.

In our observations the shock seems to occupy 1.2-1.4 arcsec. The fact that we do not observe a jump over a smaller radial range is likely to be caused by smearing effects introduced by seeing. Finally, it is important to recall that this result is based on some assumptions that must be considered carefully. In particular the density inside the flux tube, $\rho_{\mathrm{t}}$, which is obtained through the gas pressure and temperature by applying the ideal gas equation (see Sect. 2), does not satisfy vertical hydrostatic equilibrium. The Alfvén speed, and therefore the tube's critical speed, are affected by any inaccuracies in the density, which introduces some uncertainties.

\section{Summary and conclusions}

We have presented the first full inversions of infrared penumbral spectropolarimetric data with a model that accounts for the vertical and horizontal inhomogeneities of the penumbral fine structure. This model is a slightly modified version of the uncombed model of Solanki \& Montavon (1993; cf. Martínez Pillet 2000) and allows for the presence of a randomly orientated flux tube embedded in a surrounding atmosphere that harbours a strong magnetic field. The main advantages of this model, as compared to those used in Paper I (see also Mathew et al. 2003; Bellot Rubio et al. 2003) are: a) it contains two different atmospheres in the direction perpendicular to the observer (flux tube and magnetic surrounding) which allows the observed multilobed Stokes $V$ profiles to be easily reproduced; b) these two atmospheres are also present in the direction parallel to the observer line-of-sight and therefore the model includes gradients along the line of sight (in the form of sharp jumps in the physical quantities at the flux tube's boundaries) needed to produce asymmetric Stokes $V$ profiles $(\delta A \neq 0)$; c) flux tubes and their magnetic surroundings are coupled to each other using total pressure balance at all heights; d) the current model requires fewer free parameters to reproduce the data.

Feature $\mathbf{b}$ makes this model suitable to investigate the vertical size of the penumbral filaments, since the radius of the flux tube $R_{\mathrm{t}}$, which is ultimately linked to the amount of area asymmetry generated, is also obtained from the inversion. The small amount of $\delta A$ shown by the Fe I lines at $1.56 \mu \mathrm{m}$ is not enough to constrain the position of the flux tubes' upper and lower boundaries, except at the outer penumbra where $\delta A$ is sufficiently large, allowing us to set a rather accurate position for the lower boundary of the flux tube at around $\log \tau_{5} \in[-0.5,0]$, while the upper boundary remains undetected. Although for individual Stokes $V$ profiles the area asymmetry produced by the uncombed model matches the observed one only relatively inaccurately, general trends such as the radial behaviour of $\delta A$ are reproduced fairly well. We point out, in agreement with Paper I, that the use of lines that show a much larger $\delta A$ should help in this matter (see Borrero et al. in preparation).

Feature $\mathbf{c}$ has allowed us to detect, for the first time, a strong radial decrease in the pressure difference between the flux tube and its surroundings that is likely to induce an outward directed flow. This is to our mind the strongest evidence so far supporting siphon flows (Meyer \& Schmidt 1968; Montesinos \& Thomas 1993, 1997) as the physical mechanism driving the Evershed effect.

In addition, we have seen that the Evershed flow already carries velocities as large as $v \sim 4 \mathrm{~km} \mathrm{~s}^{-1}$ in the inner penumbra. This has passed unnoticed in previous works (see Schmidt \& Schlichenmaier 2000; Tritschler et al. 2004) where only Stokes $I$ was considered. A possible explanation is found in the small filling factor and inclinations, larger than $90 \mathrm{deg}$ with respect to the observer, of the magnetic field vector in the flow channels at the inner penumbra. This geometry produces small line asymmetries in Stokes $I$, but large Stokes $V$ zero crossing shifts. At the inner penumbra these fast flows are also associated with hot gas and flux tubes that are somewhat inclined with respect to the horizontal, in agreement with Schmidt \& Schlichenmaier (2000) and Rimmele (2004). These results are in close agreement with the dynamical simulations of penumbral flux tubes by Schlichenmaier et al. (1998a,b) and Schlichenmaier (2002). The tubes reach the same temperature as their surroundings very rapidly in the radial direction, and at the same time the flow speed increases smoothly (up to $v \sim 5 \mathrm{~km} \mathrm{~s}^{-1}$ ) as the pressure drops (Montesinos \& Thomas 1993, 1997).

At large radial distances the flow speed suffers a sudden decrease that is associated with positions where the tubes return to the solar interior (Westendorp Plaza et al. 1997). In addition to this well established result, we have also detected a possible transition between critical and subcritical velocities (as predicted by Montesinos \& Thomas) that is co-spatial with a rise in temperature and equivalent width at the outer penumbra, and seems to indicate that part of the kinetic energy is being dissipated into thermal energy. Therefore, part of the sudden drop in the velocity at the outer penumbra could be ascribed to the development of shock fronts.

Acknowledgements. We thank the referees, Drs. J. Thomas and B. Montesinos for valuable comments and suggestions.

\section{References}

Abrams, M. C., Davis, S. P., Rao, M. L. P., Engleman, R., Jr., \& Brault, J. W. 1994, ApJS, 93, 351

Balthasar, H. 1985, SoPh., 99, 31

Barklem, P. S., \& O'Mara, B. J. 1997, MNRAS, 290, 102

Beckers, J. M., \& Scröter, E. H. 1969, SoPh., 7, 22

Bellot Rubio, L. R. 2003, in Third International Workshop on Solar Polarization, ed. J. Trujillo Bueno, \& J. Sánchez Almeida, ASP Conf. Ser., 307, 301 
Bellot Rubio, L. R., Ruiz Cobo, B., \& Collados, M. 1998, ApJ, 506, 805

Bellot Rubio, L. R., Collados, M., Ruiz Cobo, B., \& Rodríguez Hidalgo, I. 2000, ApJ, 534, 989

Bellot Rubio, L. R., Collados, M., Ruiz Cobo, B., \& Rodríguez Hidalgo, I. 2002, Il Nuovo Cimento, 25C, 543

Bellot Rubio, L. R., Balthasar, H., Collados, M., \& Schlichenmaier, R. 2003, A\&A, 403, L47

Bellot Rubio, L. R., Balthasar, H., \& Collados, M. 2004, A\&A, 427, 319

Berdyugina, S. V., \& Solanki, S. K. 2002, A\&A, 385, 701

Börner, P., \& Kneer, F. 1992, A\&A, 260, 411

Borrero, J. M., \& Bellot Rubio, L. R. 2002, A\&A, 385, 1056

Borrero, J. M., Lagg, A., Solanki, S. K., et al. 2003a, in High Resolution Solar Observations: preparing for ATST, ed. A. Pestov, \& H. Uitenbroek, ASP Conf. Ser., 286, 235

Borrero, J. M., Bellot Rubio, L. R., Barklem, P. S., \& del Toro Iniesta, J. C. 2003b, A\&A, 404, 749

Borrero, J. M., Solanki, S. K., Bellot Rubio, L. R., Lagg, A., \& Mathew, S. K. 2004, A\&A, 422, 1093, Paper I

Bünte, M., \& Solanki, S. K. 1995, A\&A, 297, 861

Collados, M. 2001, in Advanced Solar Polarimetry: Theory, Observations and Instrumentation, ed. M. Sigwarth, ASP Conf. Ser., 236, 255

Degenhardt, D. 1989, A\&A, 222, 297

Degenhardt, D. 1991, A\&A, 248, 637

Degenhardt, D., \& Wiehr, E. 1991, A\&A, 252, 821

Degenhardt, D., \& Kneer, F. 1992, A\&A, 260, 411

Degenhardt, D., Solanki, S. K., Montesinons, B., \& Thomas, J. H. 1993, A\&A, 279, L29

Evershed, J. 1909, MNRAS, 69, 45

Ferriz-Mas, A. 1988, PhFl., 31, 2583

Frutiger, C., Solanki, S. K., Fligge, M., \& Bruls, J. H. M. J. 1999, in Solar Polarization workshop, ed. K. N. Nagendra, \& J. O. Stenflo (Astrophysiscs and Space Science library), 243, 281

Frutiger, C. 2000, Ph.D. Thesis, Institute of Astronomy, ETH, Zürich, No. 13896

Gray, D. F. 1992, The observations and analysis of stellar photospheres (Cambridge: Cambridge University Press)

Grossmann-Doerth, U., Schüssler, M., \& Solanki, S. K. 1989, A\&A, 221,338

Jahn, K. 1989, A\&A, 222, 264

Keppens, R., \& Martínez Pillet, V. 1996, A\&A, 316, 229

Kippenhahn, R., \& Möllenhoff, C. 1975, Elementare Plasmaphysik (Zürich: Wissenschaftsverlag)

Landolfi, M., \& Landi degl'Innocenti, E. 1996, SoPh., 164, 191

Lites, B. W., \& Skumanich, A. 1990, ApJ, 348, 747

Lites, B. W., Elmore, D. F., Seagraves, P., \& Skumanich, A. P. 1993, ApJ, 418, 928

Livingston, W., \& Wallace, L. 1991, An Atlas of the Solar Spectrum in the infrared from 1.1 to 5.4 microns, NSO Technical Rep., 91

Martínez Pillet, V. 2000, A\&A, 361, 734

Martínez Pillet, V. 2001, A\&A, 369, 644

Martínez Pillet, V., Collados, M., Bellot Rubio, L. R., et al. 1999, AGM, 15, P05

Mathew, S., Lagg, A., Solanki, S. K., et al. 2003, A\&A, 403, 695

Mathew, S. K., Lagg, A., Solanki, S. K., et al. 2004, A\&A, 422, 703

McPherson, M. R., Lin, H., \& Kuhn, K. R. 1992, SoPh., 139, 255

Meyer, F., \& Schmidt, H. U. 1968, Z. Ang. Math. Mech, 48, T218

Mies, F. H. 1974, J. Molec. Spec., 53, 150

Montesinos, B., \& Thomas, J. 1997, Nature, 390, 485

Müller, D. A. N., Schlichenmaier, R., Steiner, O., \& Stix, M. 2002, A\&A, 393, 305
Penn, M. J., Cao, W. D., Walton, S. R., Chapman, G. A., \& Livingston, W. 2003, ApJ, 590, L119

Press, W. H., Flannery, B. P., Teukolsky, S. A., \& Vetterling, W. T. 1986, Numerical Recipes (Cambridge: Cambridge University Press)

Rimmele, T. 1995, A\&A, 298, 260

Rimmele, T. 2004, ApJ, 604, 906

Rouppe van der Voort, L., Löfdahl, M. G., Kiselman, D., \& Scharmer, G. B. 2004, A\&A, 414, 717

Rüedi, I., Solanki, S. K., Keller, C. U., \& Frutiger, C. 1998, A\&A, 338, 1089

Rüedi, I., Solanki, S. K., \& Keller, C. U. 1999, A\&A, 348, L37

Ruiz Cobo, B., \& Del Toro Iniesta, J. C. 1992, ApJ, 398, 375

Sánchez Almeida, J. 1998, ApJ, 497, 967

Sánchez Almeida, J. 2001, A\&A, 369, 643

Sánchez Almeida, J., \& Lites, B. W. 1992, ApJ, 398, 359

Scharmer, G., Gudiksen, B. V., Kiselman, D., et al. 2002, Nature, 420, 151

Schlichenmaier, R. 2002, AN, 323, 303

Schlichenmaier, R., \& Collados, M. 2002, A\&A, 381, 668

Schlichenmaier, R., Jahn, K., \& Schmidt, H. U. 1998a, A\&A, 337, 897

Schlichenmaier, R., Jahn, K., \& Schmidt, H. U. 1998b, ApJ, 483, L121

Schlichenmaier, R., Müller, D. A. N., Steiner, O., \& Stix, M. 2002, A\&A, 381, L77

Schmidt, W., \& Schlichenmaier, R. 2000, A\&A, 364, 829

Solanki, S. K. 2003, A\&ARv, 11, 153

Solanki, S. K., \& Rüedi, I. 2003, A\&A, 411, 249

Solanki, S. K., \& Montavon, C. A. P. 1993, A\&A, 275, 283

Solanki, S. K., Rüedi, I., \& Livingston, W. 1992, A\&A, 263, 312

Solanki, S. K., Montavon, C. A. P., \& Livingston, W. 1994, A\&A, 283, 221

Solanki, S. K., Rüedi, I., Bianda, M., \& Steffen, M. 1996, A\&A, 308, 623

Solanki, S. K., Finsterle, W., Rüedi, I., \& Livingston, W. 1999, A\&A, 347, L27

Stanchfield, D. C. H., Thomas, J. H., \& Lites, B. W. 1997, ApJ, 477, 485

Stellmacher, G., \& Wiehr, E. 1980, A\&A, 82, 157

Sütterlin, P. 2001, A\&A, 374, L21

Sütterlin, P., Bellot Rubio, L. R., \& Schlichenmaier, R. 2004, A\&A, 424, 1049

Title, A., Frank, Z., Shine, R., et al. 1993, ApJ, 403, 780

Thomas, J. H. 1988, ApJ, 333, 407

Thomas, J., \& Montesinos, B. 1991, ApJ, 375, 404

Thomas, J., \& Montesinos, B. 1993, ApJ, 407, 398

Thomas, J., \& Weiss, N. 2004, A\&ARv, 42, 517

del Toro Iniesta, J. C., Tarbell, T. D., \& Ruiz Cobo, B. 1994, ApJ, 436, 400

del Toro Iniesta, J. C., Bellot Rubio, L. R., \& Collados, M. 2001, ApJ, 549, L139

Tritschler, A., Schlichenmaier, R., Bellot Rubio, L. R., \& KAOS Team 2004, A\&A, 415, 717

Wentzel, D. G. 1992, ApJ, 388, 211

Westendorp Plaza, C., del Toro Iniesta, J. C., Ruiz Cobo, B., et al. 1997, Nature, 389, 47

Westendorp Plaza, C., del Toro Iniesta, J. C., Ruiz Cobo, B., \& Martínez Pillet, V. 2001a, ApJ, 547, 1148

Westendorp Plaza, C., del Toro Iniesta, J. C., Ruiz Cobo, B., et al. 2001b, ApJ, 547, 1130

Wiehr, E. 1995, A\&A, 298, L17

Wiehr, E. 1997, in Advances in Physics of Sunspots, ed. B. Schmieder, J. C. del Toro Iniesta, \& M. Vázquez, ASP Conf. Ser., 118, 175

Wiehr, E., Stellmacher, G., Knölker, M., \& Grosser, H. 1986, A\&A, 155,402

Wittmann, A. 1974, SoPh, 35, 11 